CASE STUDY: Monsters, Microbiology and Mathematics: the epidemiology of a zombie apocalypse

J.Verran ${ }^{1 *}$, M.Crossley ${ }^{2}$, K.Carolan $^{1}$, N.Jacobs ${ }^{2}$, M.Amos $^{2}$,

${ }^{1}$ School of Healthcare Science and ${ }^{2}$ School of Computing, Mathematics and Digital

Technology, Manchester Metropolitan University, Chester St., Manchester M1 5GD.

Running header: Epidemiology of zombie apocalypse

Key words: epidemiology - mathematical modelling - zombies

Corresponding author: Prof Joanna Verran, School of Healthcare Science, Manchester Metropolitan University, Chester St., Manchester M1 5GD.

Tel: 00-44-161-2471206

j.verran@mmu.ac.uk 


\section{CASE STUDY: Monsters, Microbiology and Mathematics: the epidemiology of a zombie}

apocalypse

\section{Abstract}

The aim of this learning exercise was to harness current interest in zombies in order to educate audiences about the epidemiology of infectious disease. Participants in the activity were provided with an outbreak scenario, which they then used as the basis of play-based activities. By considering the mode and speed of transmission, size of outbreak, and prevention/control strategy, participant groups were able to define parameters of their outbreak scenario. These were then input to SimZombie, a computer simulation program developed by the authors, which visually demonstrated the spread of infection through a population. The resulting animations were then used as the basis of in-depth discussion, which, in turn, enabled the consideration of principles of disease transmission and control strategies.

The activity provided an opportunity to engage a range of audiences through a variety of different delivery mechanisms, including role play, workshop and informal drop-in. Learning was evidenced by participation and feedback.

\section{Introduction}

Given the increasingly connected nature of the global community, there is a pressing need for a better public understanding of disease dynamics (Hufnagel, et al.; 2004). In UK schools and colleges, knowledge of infectious diseases is indicated in the school science curriculum (Department for Education, 2013). For the general public, salient facts about infectious disease include principles of disease prevention and control and awareness of transmission routes. Public information is generally provided through the media, leaflets in 
surgeries/pharmacies and via the internet, but these are typically "one-way" modes of communication, with no subsequent evaluation of learning acquisition (Bemelmans, 2007). Engagement can therefore be enhanced by modifying the delivery system to use a novel vehicle or context, such as mixing science with art, literature (Verran 2013a) or games (Michael and Chen, 2005).

Vampires, and other 'monsters' such as zombies and werewolves, potentially offer a focus for engagement with disease transmission and outbreaks. Thus, a workshop delivered for the 2010 Manchester Children's Book Festival (MCBF: Verran, 2010, 2013b) used the Twilight novel (Stephenie Meyer, 2005; movie 2008, directed by Catherine Hardwick) in that context. The workshop was set in a Biology laboratory, with microscopes and slides of the cell cycle available for examination. Using readings from the novel and facilitated discussion, the participants identified routes of vampirism/disease transmission, and considered analogous methods of prevention (garlic as a representation of an antimicrobial; 'not inviting them in' representing behaviour modification).

Meanwhile, an agent-based simulation tool had been developed by the second author, as part of his undergraduate studies (Crossley and Amos 2011; software available at https://code.google.com/p/simzombie/). The program (SimZombie) simulates the spread of infection through an animated population of individuals, using an individual-based version of a standard susceptible/infected/recovered model (Munz, et al.; 2009), and graphically depicts different categories of individual over time (Figure 1).

Different patterns of disease spread emerge, depending on the parameter values entered into the programme. Using our 'monster' analogy, zombie infections are slow to spread, but inexorable; vampire outbreaks spread quickly, but are less efficient; werewolves are only active once in a lunar month, thus the infection goes 'latent' with clusters emerging in 
different location over time. Animations of all of these outbreaks are available for download at http://dx.doi.org/10.6084/m9.figshare.729232

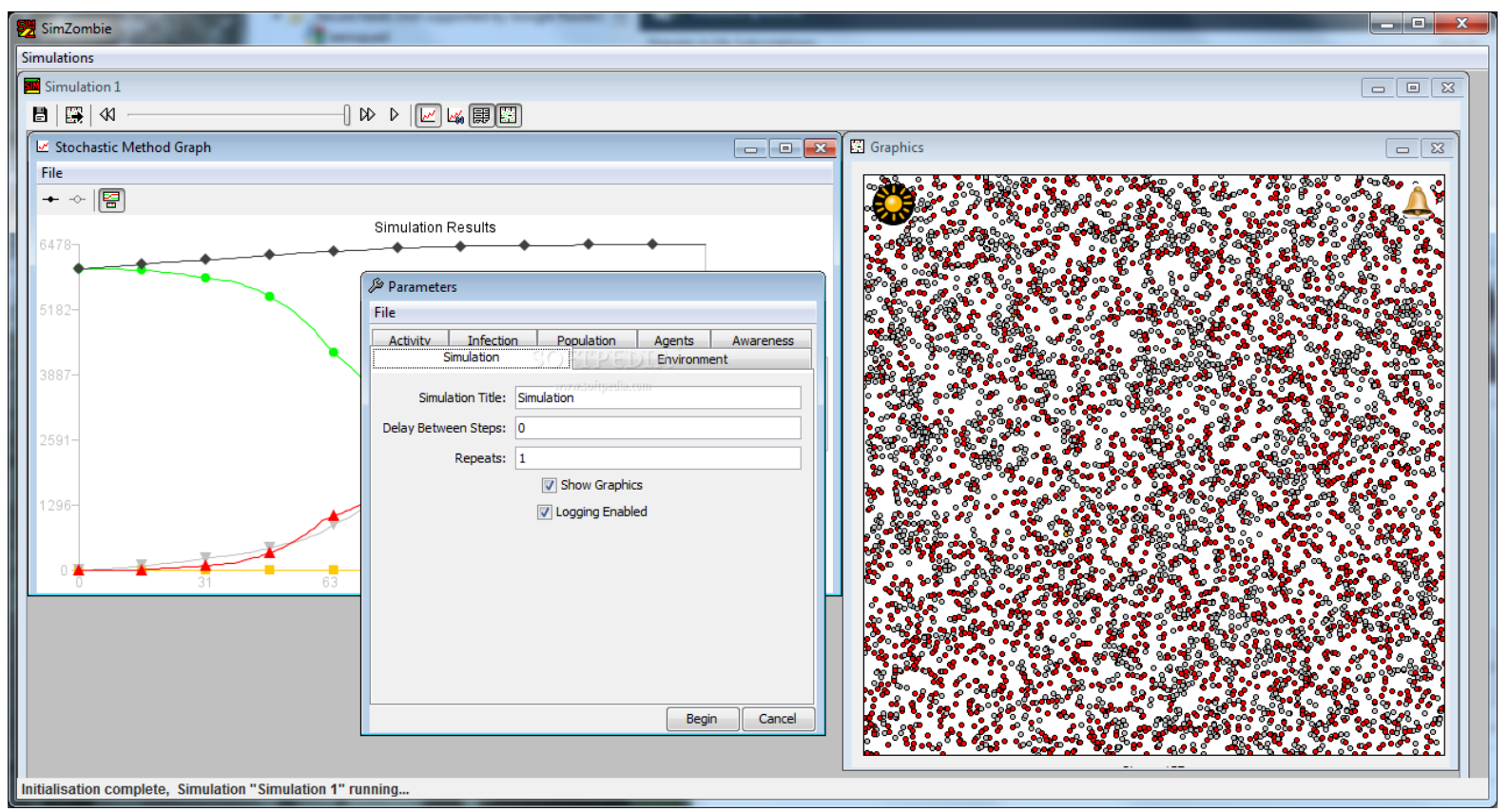

Figure 1: Sample screenshot of SimZombie program.

The Monsters, Microbiology and Maths (MoMiMa) group at Manchester Metropolitan University was formed in 2010, comprising individuals with a common interest in 'monsters'. The group's aim was to integrate this expertise and enthusiasm, and SimZombie, into public engagement activities. The rest of this paper describes the development of these activities, in order to facilitate engagement with a range of audiences. The main events, processes, deliverables, outcomes and and feedback mechanisms are summarised in Figure 2. We now present our work in the context of the various stages of its evolution. 
Figure 2: Flow chart depicting the evolution of the project.

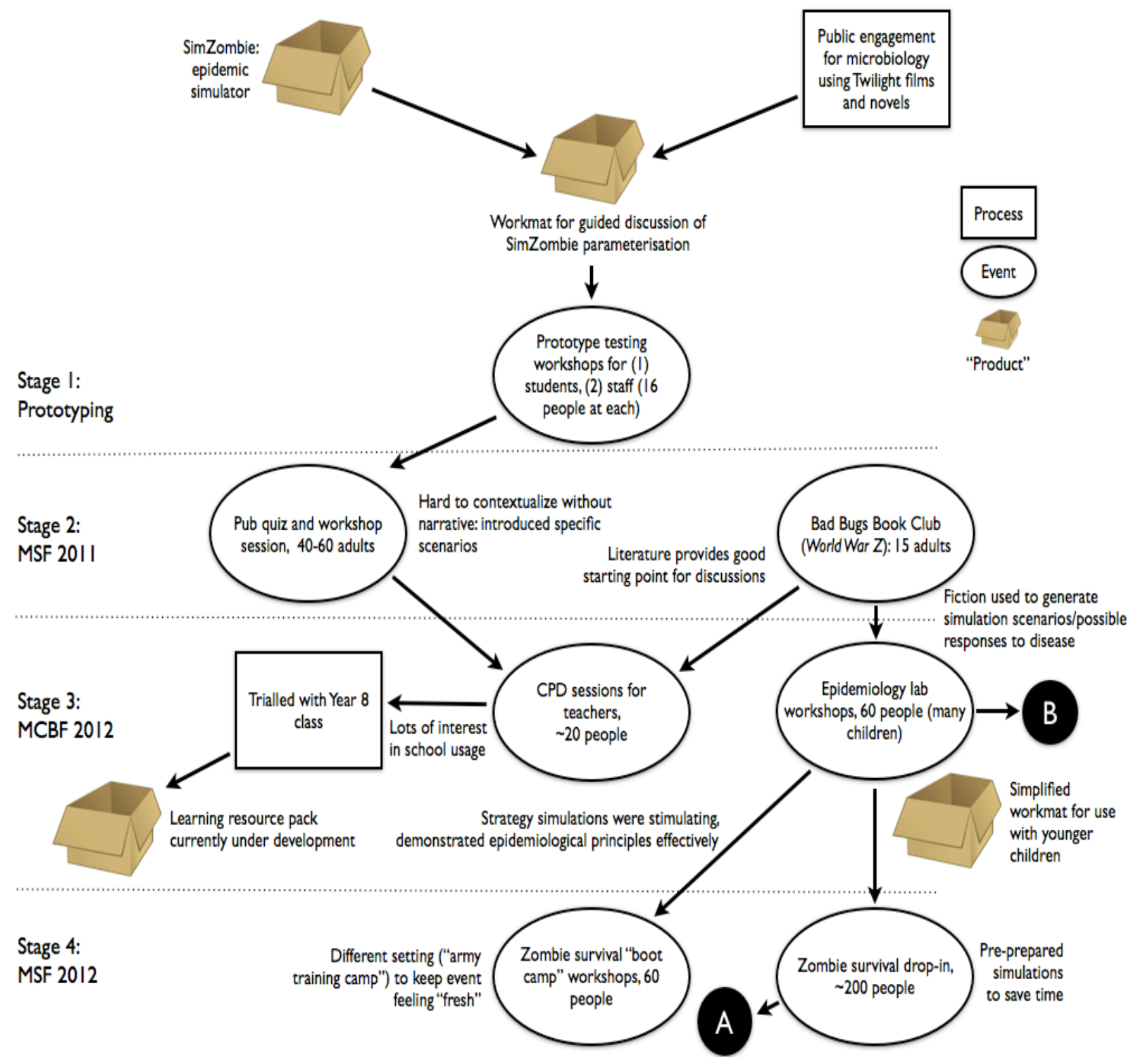




\section{Stage 5:}

Saturday Science,

MOSI 2013

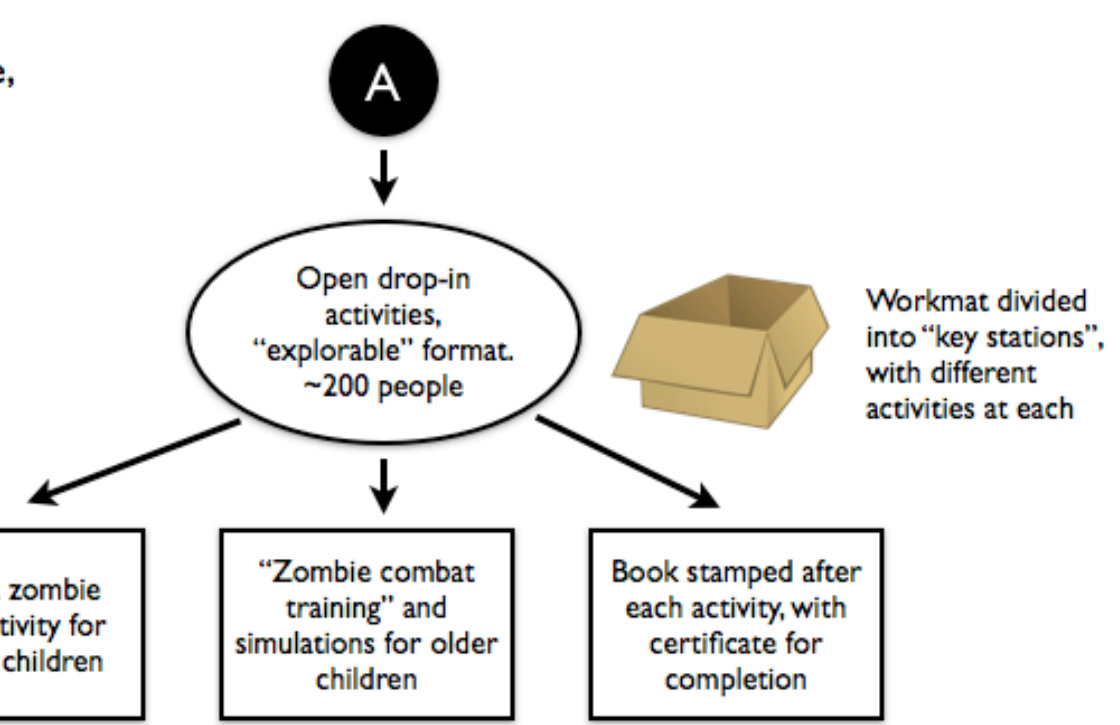

Stage 6:

Deadinburgh,

2013

\section{B}

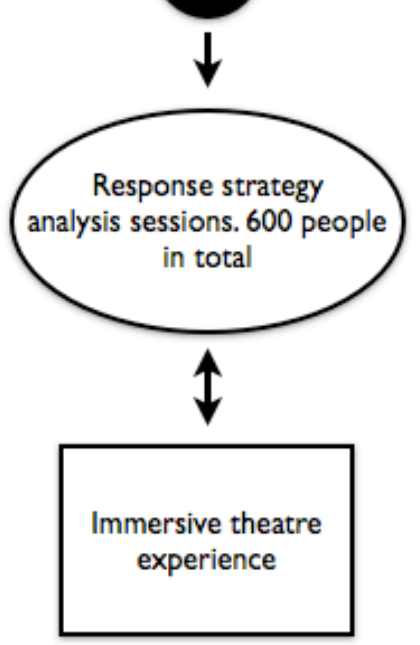

Figure 2: Flow chart depicting the evolution of the project.

\section{Stage 1: Development of materials around SimZombie}

Additional key characteristics to differentiate the kinetics of outbreaks of vampires, zombies and werewolves were identified. For example, vampires move quickly, but are only active at night; victims may die, or become vampires (or vampire 'food'); zombies are active all the time, but move slowly (the exception being the recently emerging 'zoombies' (Botting, 
2010)), and transmission is $100 \%$ effective and $100 \%$ 'lethal', unless the victim is consumed. These fictional characteristics can be used to parallel true disease outbreaks- for example, the relative transmission and infection rates of H5N1 (bird flu - for man, low transmission rate but high virulence), or the recent H1N1 pandemic (swine flu - high transmission rate but low virulence); the latency of herpes or HIV; the duration of incubation periods and impact on epidemiology (for example chronic diseases such as tuberculosis, or acute infections such as measles); the high virulence of Ebola but lack of airborne transmission; and so on (Verran, 2013c).

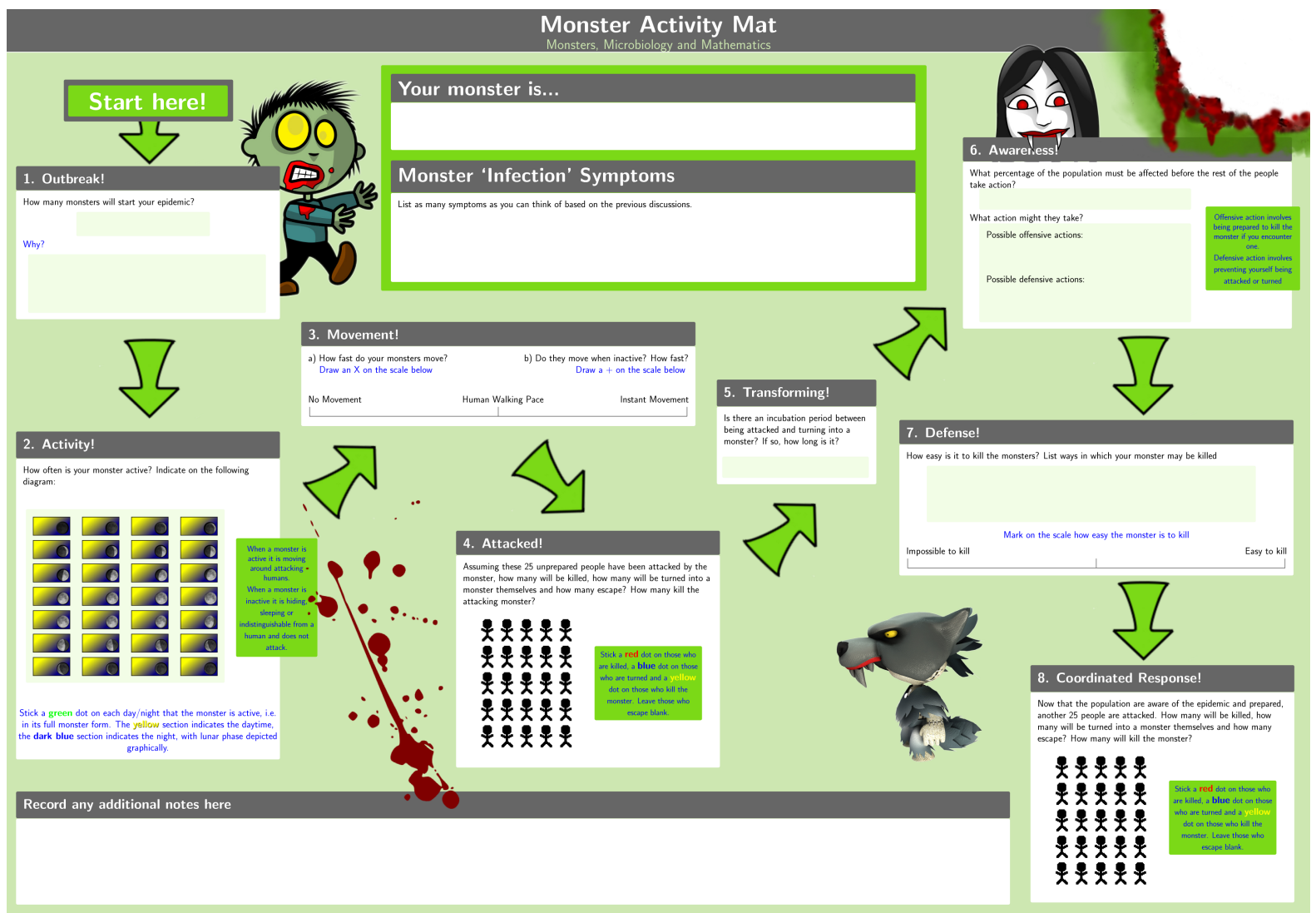

Figure 3: Activity workmat. 
An A1-size activity workmat (Figure 3) was developed to encourage exploration of selected disease parameters. The mat was initially trialled by focus groups, comprising staff and students from a range of disciplines across the University. Questions on the mat prompted participants to consider the parameters of a zombie, vampire or werewolf outbreak scenario, derived from literature, imagination, film or game. Responses were subsequently used as parameter values, which were fed into the SimZombie program. The resultant output presented a visual illustration of the given outbreak. Although the discussions and subsequent simulation visualisations were useful, it was clear that participants experienced difficulties in contextualising the outbreaks without an accompanying narrative. Thus, specific 'scene-settings' were developed, with a particular focus on zombie outbreaks, due to the perceived interest of both genders in zombies (Higson, 2013).

\section{Stage 2: First public delivery of materials}

MoMiMa offered a series of events at the 2011 Manchester Science Festival (MSF), each targeted at a different audience. At an evening 'pub quiz' (Kappa, 2009), ten teams of 4-6 people were tasked with answering questions on monsters (including a 'film clips' round), microbiology (including a 'match the disease to the microbe' picture round) and general scientific knowledge. In between rounds, each team used an activity mat to identify parameter values associated with a particular outbreak scenario (a short descriptive paragraph on a card). During the marking of the quiz rounds, the simulations resulting from their parameter values were presented, yielding a rich collection of varied 'outbreaks' at the end of the evening. These enabled discussions centred on the progression of various outbreaks. Feedback was acquired via a short questionnaire, in which participants were asked to rank enjoyment, organisation, learning of science and likelihood of attending a 
repeat event, on a 1 - 5 scale. Free text comments were also sought. From 24 respondents, the average scores for enjoyment, organisation, learning and repeat attendance were 4.5, 4.1, 3.5 and 4.5 respectively. Text comments were positive overall; negative comments alluded to venue-specific issues such as noise and lack of space, the difficulty of the microbe quiz (two comments), and a request for more information on the simulations (two comments). The relative perceived lack of 'science learning' in comparison to other scores was slightly disappointing: more overt emphasis on epidemiology principles might be made in future.

\section{Stage 3: Using fiction to enhance engagement}

At MSF 2011, the Bad Bugs Bookclub (Verran, 2013c) hosted complementary events where World War Z (Max Brooks, 2006), and I am Legend (Richard Matheson, 1954) - both apocalyptic zombie/vampire novels - were discussed. Like the activity workmat, the books (and movie screenings of three versions of I am Legend) stimulated discussion on disease epidemiology.

Well over 200 individuals were reached by these activities. The combination of literature, computer program and disease epidemiology was then developed for the 2012 Manchester Children's Book Festival. The aim of this festival is to encourage the engagement of children with literature. Thus, four 'zombie novels' aimed at a teenage audience (Boneshaker by Cherie Priest [2009], Warm Bodies by Isaac Marion [2011], Rot and Ruin by Jonathon Maberry [2010], and The Dead by Charlie Higson [2010]) were selected for incorporation into a workshop held at the Festival Family Fun Day. Participants signed up to the workshop at a reception desk where media make-up artists 'zombified' visitors throughout the day. 
Short summaries of each book were provided to (four) facilitators, along with a brief outbreak scenario. The facilitators were role-playing as epidemiologists, and participants became scientists working in an epidemiology laboratory. The presented scenario was that of a raging pandemic, and participants urgently needed to identify the parameters of the outbreak with a view to prevention and control. Simulated news broadcasts and Twitter feeds contributed to the atmosphere. Participants (predominantly families or teenagers) were grouped at one of four tables, each focusing on a different novel. Facilitators read excerpts from the novels, and the groups identified parameters of their outbreaks using a simplified workmat design (full versions of all workmats are downloadable from http://dx.doi.org/10.6084/m9.figshare.729232). In all of the novels, it became apparent that the strategy for control was to isolate the uninfected (analogous to the use of immunisation to protect populations) or the infected (quarantine). SimZombie was modified to depict these response strategies of population enclosure, and limitations of the isolation of the uninfected strategy were graphically demonstrated, where the zombies were able to enter the 'safe' enclave through a break in the perimeter walls. This was extrapolated to discussion on the importance of high vaccine uptake levels to ensure herd immunity. Quarantine of the infected was successful if the boundary walls were strong, and if numbers of uninfected individuals were high.

The 45-minute workshop was run three times, with 20 participants in each workshop. For feedback, before they left, participants were asked to note (on post-it notes) what they enjoyed, and/or what they had learned. Of twenty-seven responses, 25 were positive, with specific references made to microbiology (9 responses) and zombies (11 responses), such as 'I learnt how difficult it would be to control a very serious disease', 'how athletes can be more prone to disease' and 'I learnt how disease spreads'. The make-up sessions were 
especially popular, but somewhat distracting. The activity would also have been enhanced if participants had read the books in advance of the event.

\section{Stages 4-6: Time constrained delivery of key messages}

The response strategy simulations proved valuable for demonstrating principles of epidemiology, and encouraging discussion. These key messages were carried forward to the 2012 Manchester Science Festival and delivered via an army-style 'bootcamp' event, focusing on how to deal with a zombie outbreak. The sessions began with a structured (Powerpoint) presentation, which included background information on the workshop followed by an interactive session. Each participant was issued with a handbook guide, which was used as a trigger for discussion (epidemiology, disease transmission, prevention and control) around previously prepared outbreak simulations. This format ensured delivery of key scientific messages to the entire group, and reduced the number of facilitators required. Participants were awarded a certificate at the end of the event. The three 45-minute workshops each attracted around 20 participants, and again feedback was positive ( 22 positive responses of 23 ), with comments demonstrating improved acquisition of understanding. For example: 'simulations were excellent for showing how infections can spread'; 'like the simulations. The difference between quarantine and isolation was really striking'; '(name) is studying the plague, so really good at showing infection prevention and survival'; 'great way to explain infection to kids. Well presented. Clear and fun'.

Despite the success of these activities, they were time-consuming in terms of audience numbers, and higher throughput delivery would be desirable for a future event. 
Saturday Science events are delivered monthly by visiting scientists at the Manchester Museum of Science and Industry (http://www.mosi.org.uk/), and the zombie activity was modified again to enable a 'drop-in' style delivery of the outbreak simulation at one of these events in 2013. Only SimZombie pre-prepared simulations were used at this event, and the workmat was altered to a 'key questions' format - each question becoming a 'station' for visitors to attend (in any sequence). The questions addressed (i) Movement: how fast a disease is able to spread geographically, (ii) Survival: which response strategies the participants might consider, and why, (iii) Transmission: how do people become zombies, and what other transmission methods exist for other diseases? A station for younger children asked 'What do viruses look like?' Participants looked at images of viruses, and then drew their own zombie virus. Older children took part in target practice (on a life size zombie dummy) using toy weaponry. The effect of their 'hit' was noted - for example, congratulations were offered to those managing to hit the head (inactivates zombies). An accompanying booklet posed key questions to be answered at each stage of the activity. Visitors were then given the opportunity to present a completed booklet to the bootcamp 'corporals', in order to become certified 'zombie survival experts'.

This activity reached a larger audience (an estimated number of visitors totalled around 150 over a 4 hour period), but was not without drawbacks. A larger workforce was required (STEM ambassadors), because each station needed to be manned by an expert. Due to the drop-in nature of the event, it was not easy to obtain feedback: nineteen responses noted enjoyment, but lacked specific comments related to the subject. A question and answer session with STEM ambassadors and event organisers yielded more constructive comments regarding event organisation. It was not easy to encourage visitors to participate in all of the station activities - perhaps a stricter 'follow the path' layout may have encouraged this. 
It was also less apparent that significant learning had taken place - although engagement was evident.

Finally, 'Deadinburgh' was an immersive event delivered by LAS theatre group in April 2013. The old Royal Edinburgh Veterinary Hospital provided the location for a 'safe zone' where scientists were working on a cure for the 'Lazarus Pathogen' (LP) outbreak which had caused Edinburgh to be isolated. Over four nights, 1000 members of the public entered the safe zone, chased by infected "PALPs" (persons affected with the Lazarus Pathogen) (actors) and herded by soldiers (also actors) into the laboratories. Scientists (not actors) were unable to agree on a strategy from the three choices available: to continue the quarantine and wait for a cure; cull the infected; sacrifice the city by annihilation with an explosive agent. The audience was therefore required to listen to the scientists and make the decision themselves. The result of a vote would determine the fate of the city. As one of the six scientist groups, the MoMiMa team (of two) used the epidemiology laboratory scenario, delivered to three groups of 40 adults within a 20 minute period, for four nights. The highly structured activity involved an overview of the science behind the outbreak and the simulation, followed by five minute group discussions on the choices available, and identification of appropriate outbreak parameters. All simulations were presented in order to help participants to make their final decision based on all of the scientific information available to them. Data and discussion derived from the exercises emphasised the importance of: maintaining a strong boundary when separating the infected from the uninfected (immunisation); knowing the incubation period of the infection; the carrier state; diagnosing infections with accuracy and precision; ensuring that the control or prevention strategy is effective. This project was supported by the UK BBSRC, and project evaluation is ongoing. A four-star review of the event in The Scotsman (April 22, 2013) stated that 
“...while there's plenty of talk about bridging the gap between arts and science, this is a show that actually does it; and provides a good, exhausting, thought-provoking night out into the bargain."

\section{Conclusions and future work}

SimZombie and its associated activities have engaged a wide range of audiences through a number of different delivery events: University staff and students, adults, families and young people. An overview of the activities was delivered at a teacher CPD session, and was very well received. The versatility of the activity was commended, particularly the potential for cross-subject work (literature, microbiology, mathematics). A year 8 class were similarly enthused. For schools, key learning outcomes regarding disease epidemiology, using zombies as the vehicle of infection, can be demonstrated and explored in an engaging and unusual context. Issues of infection and behaviour can be considered in PSHE (personal, social, health and economic studies)-related activities. In biology courses, SimZombie can be used to demonstrate the epidemiology of real disease outbreaks. In addition to the materials made available through this publication, we are currently developing a school resource pack, to include information on disease epidemiology, copies (paper and electronic) of workmats, and an accessible and interactive version of SimZombie. This will remove the need for direct coordination by MoMiMa, and increase the reach and impact of activities. 


\section{Acknowledgements}

MoMiMa wish to acknowledge the support of the EPSRC Bridging the Gaps: NanolnfoBio project (EP/H000291/1) and the Society for Applied Microbiology (www.sfam.org.uk) for providing financial support for some of the events. HEA National Teaching Fellowship funding awarded to JV was also used to provide support.

\section{References}

Bemelmans, M. (2007).“Carrots, Sticks, and Sermons: Policy Instruments and Their Evaluation". New Brunswick, NJ, Transaction Publishers.

Botting, F. (2010). "Zoombie London: unexceptionalities of the new world order". In London Gothic: Place, Space and the Gothic Imagination, edited by L. Phillips and A. Witchard, pp. 153-170. London, UK. Continuum, 2010.

Crossley, M. and Amos, M. (2011)."SimZombie: a case-study in agent-based simulation construction". In Agent and Multi-Agent Systems: Technologies and Applications, Lecture Notes in Artificial Intelligence (LNAI). 6682, pp. 514-523.

Department for Education (2013) "Science : Programme of study for Key Stage 4". February 2013, draft programme available at http://bit.ly/14synjE.

Higson, C. 2013. The rise of the zombie. Radio Times magazine, 16- 22 March, p30-31. Hufnagel, L., Brockmann, D. and Geisel, T. (2004) “Forecast and control of epidemics in a globalized world". Proceedings of the National Academy of Sciences of the USA 101(42), 15124-15129.

Klappa, P. (2009). “Promoting active learning through 'pub quizzes' - a case study at the University of Kent. Bioscience Education 14: DOI: 10.3108/beej.14.c2 
Michael, D.R. and Chen, S.L. (2005). "Serious Games: Games that Educate, Train and Inform." Muska and Lipman.

Munz, P., Hudea, I., Imad, J., Smith?, R. (2009)“When zombies attack!: Mathematical modelling of an outbreak of zombie infection". In Infectious Disease Modelling Research Progress, ed. JM Tchuenche and C. Chiyaka, Hauppauge NY: Nova Science Publishers pp. 133-150.

Verran J. (2010). "The microbiology of vampires".Microbiology Today 37 (4): 248 - 9.

Verran J. (2012). “Monsters, Maths and Microbiology". Microbiologist 13 (1).43 - 44.

Verran J. (2013a). "Mixed cultures: microbiology, art and literature". In For the Love of Learning, edited by T. D. Bilham. In Press. Palgrave McMillan.

Verran J. (2013b). "Case studies in public engagement". In Science Communication: a practical guide for scientists edited by L. Bowater and K. Yeoman, pp. 144-145 and 206-208. Wiley Blackwell.

Verran J. (2013c). "The Bad Bugs Bookclub: science, literacy and enjoyment". Journal of Microbiology and Biology Education 14 (1): 110 - 112

Verran J. (2013d). "The return of the zombies". Microbiologist 14 (2): $26-27$. 